\title{
Children with Comorbid Speech Sound Disorder and Specific Language Impairment are at Increased Risk for Attention-Deficit/Hyperactivity Disorder
}

\author{
Lauren M. McGrath • Christa Hutaff-Lee • \\ Ashley Scott • Richard Boada • Lawrence D. Shriberg • \\ Bruce F. Pennington
}

Published online: 20 September 2007

(C) Springer Science+Business Media, LLC 2007

\begin{abstract}
This study focuses on the comorbidity between attention-deficit/hyperactivity disorder (ADHD) symptoms and speech sound disorder (SSD). SSD is a developmental disorder characterized by speech production errors that impact intelligibility. Previous research addressing this comorbidity has typically used heterogeneous groups of speech-language disordered children. This study employed more precise speech-language diagnostic criteria and examined ADHD symptomatology in 108 SSD children between the ages of 4 and 7 years old with specific language impairment (SLI) $(n=23,14$ males, 9 females) and without SLI ( $n=85,49$ males, 36 females). We also examined whether a subcategory of SSD, persistent $(n=39$,
\end{abstract}

L. M. McGrath $(\bowtie) \cdot$ C. Hutaff-Lee · A. Scott • B. F. Pennington Department of Psychology, University of Denver,

Frontier Hall 2155 S. Race St.,

Denver, CO 80209, USA

e-mail:1mcgrath@du.edu

C. Hutaff-Lee

School of Applied Psychology and Counselor Education,

University of Northern Colorado,

Greeley, CO, USA

A. Scott

InterDepartmental Neuroscience Program,

University of California, Los Angeles,

Los Angeles, CA, USA

R. Boada

Department of Pediatrics,

University of Colorado Health Sciences Center,

Denver, CO, USA

L. D. Shriberg

Waisman Center, University of Wisconsin, Madison,

Madison, WI, USA
25 males, 14 females) versus normalized SSD $(n=67,38$ males, 29 females), was associated with ADHD and/or interacted with SLI to predict ADHD symptomatology. Results indicated that participants in the SSD + SLI group had higher rates of inattentive ADHD symptoms than those in the SSD-only and control groups. In addition, an unexpected interaction emerged such that children with SLI and normalized-SSD had significantly higher ADHD inattentive ratings than the other subgroups. A proposed explanation for this interaction is discussed.

Keywords Speech sound disorder.

Specific language impairment - Speech-language disorders · Attention-deficit/hyperactivity disorder . Comorbidity

\section{Introduction}

Comorbidity seems to be the rule rather than the exception among child and adult psychopathologies (Pennington 2002). Understanding the basis for comorbidity is an important step towards understanding the etiology of disorders. Here, we focus on a potential comorbidity between Attention-Deficit/Hyperactivity Disorder (ADHD) and Speech Sound Disorder (SSD).

SSD is a developmental disorder characterized by speech production errors that significantly impact intelligibility (Shriberg 2003). For example, children with SSD may make age-inappropriate speech sound deletions (e.g., "do" for "dog") and/or substitutions (e.g., "ti" for "see"). In the current Diagnostic and Statistical Manual of Mental Disorders (DSM-IV-TR: American Psychiatric Association (APA) 2000), the disorder is referred to as Phonological Disorder. In earlier versions of the DSM, the disorder was 
referred to as Developmental Articulation Disorder. Although the terms Phonological Disorder and SSD refer to the same constellation of clinical signs, the term SSD is currently preferred because it recognizes that the disorder may have antecedents in both articulatory (sensorimotor) and phonological (cognitive-linguistic) domains. The term SSD has recently been adopted as the classification entity for this disorder by the American Speech-LanguageHearing association.

It is particularly interesting to consider the relationship between ADHD and SSD if their respective comorbidities with reading disability $(\mathrm{RD})$ are included in the theoretical analysis. It is well-established that ADHD is comorbid with $\mathrm{RD}$ at a rate estimated at 25-40\% (Rucklidge and Tannock 2002; Willcutt and Pennington 2000) and SSD is comorbid with RD at approximately the same rate $(25-30 \%)$ (Gallagher et al. 2000; Pennington and Lefly 2001; Scarborough 1990). Recent molecular genetics analyses have heightened interest in the comorbidity of RD, ADHD, and SSD because all three disorders have shown linkage to the same genetic locus at 6p22 (Fisher and DeFries 2002; Smith et al. 2005; Willcutt et al. 2002). Additionally, several studies have identified loci that are pleiotropic for RD and ADHD (Bakker et al. 2003; Gayan et al. 2005; Loo et al. 2004) and RD and SSD (Miscimarra et al. 2007; Smith et al. 2005; Stein et al. 2004, 2006). This preliminary evidence for shared genetic risk factors suggests that all three disorders may overlap phenotypically, to some extent. The Venn diagrams in Fig. 1 illustrate two possible scenarios for the relationships between these three disorders.

An additional consideration when analyzing the overlap of RD, ADHD, and SSD is a fourth disorder, Specific Language Impairment (SLI), that is also comorbid with each disorder in the triad (e.g., Beitchman et al. 1989a; Bishop and Adams 1990; Shriberg et al. 1999). SLI is characterized by delays in language development, including difficulties with vocabulary, semantics, and/or syntax that cannot be explained by general cognitive delays, hearing
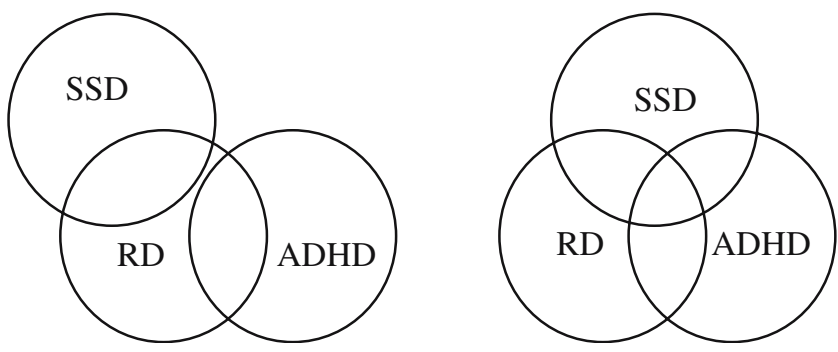

Fig. 1 Two possible scenarios for the relationship between RD, ADHD, and SSD. In the first figure, ADHD and SSD do not overlap despite their mutual relationship with RD. In the second figure, SSD and ADHD overlap with each other, as well as with RD. Note that these figures do not include chance overlap problems, neurological problems, or physical handicaps (Bishop 1997, 2006). The term SLI encompasses the DSMIV categories of Expressive Language Disorder and Mixed Receptive-Expressive Language Disorder (APA 2000).

The genetic relationship of SLI to the RD-ADHD-SSD triad of disorders is not very clear. Two independent genome-wide screens of SLI have not identified any overlapping loci with RD, SSD, or ADHD (Bartlett et al. 2002; SLI Consortium 2002, 2004). At present, it is unclear if the negative findings are due to a lack of power or a true null finding.

At this point, the potential association between ADHD and SSD has been less well-studied than the relationship of each disorder with RD. Additionally, the impact of comorbid SLI on the relationship between ADHD and SSD has been difficult to determine because studies have typically employed less precise diagnostic criteria for speech-language disorders than are used in contemporary research. The current study attempts to fill this gap in the literature by examining the severity of ADHD symptoms in a sample of children with SSD who are subgrouped according to well-defined speech-language disorder categorizations. In the following sections, we will review previous studies investigating the overlap of speechlanguage disorders and ADHD and provide a rationale for the hypotheses addressed in the current study.

\section{Previous Studies of Speech-Language Disorders and ADHD}

Several studies conducted in the 1980 s led to a consensus that speech-language impairment frequently co-occurred with ADHD in both clinic-referred (Baker and Cantwell 1982; Baker and Cantwell 1992; Cantwell and Baker 1985; Gualtieri et al. 1983; Love and Thompson 1988) and epidemiological samples (Beitchman et al. 1986, 1989a, 1990). These studies found comorbidity rates ranging from about $17-66 \%$, with an epidemiological sample showing a $30 \%$ rate of overlap (Beitchman et al. 1986). Although these studies used the diagnostic equivalents of ADHD from earlier versions of the DSM (APA 1980, 1987), there is considerable symptom overlap between the earlier definitions and the current DSM-IV-TR ADHD criteria (Morgan et al. 1996).

Advances in the definition of speech-language disorders have spurred renewed interest in the overlap of speechlanguage disorders and ADHD (e.g., Snowling et al. 2006). In previous research, groups defined as "speech and language disordered" were often quite heterogeneous and the disciplines of psychiatry, speech-language pathology, and psychology often used different operational definitions. The terms speech disorder and language disorder were 
even used interchangeably (Baker and Cantwell 1985). The division between the two types of developmental disorders is more clearly drawn in contemporary research. New classification schemes distinguish between and within speech (e.g., SSD) and language disorders (e.g., SLI). Thus, current diagnostic systems allow for a more finegrained analysis of speech-language disorders and their overlap with ADHD than was possible in previous decades.

Although most previous studies used heterogeneous groups of speech-language disordered children, one study did attempt to group children according to their speech and language profiles and determine which group was most at risk for attention deficit disorders (Beitchman et al. 1989a, b). Although their sample size was small, the investigators found that children with poor speech and language skills were at increased risk for attention problems (59\% rate of comorbidity) compared to children with speech deficits alone (5\% rate of comorbidity) (Beitchman et al. 1989a). These results may qualify the conclusion of the previous studies that speech-language disorders are universally associated with attention deficit disorders.

\section{Current Study}

The intent of the current study was to replicate the results of Beitchman et al. (1989a) and extend the conclusions by considering speech and language subgroups within the SSD sample. We recruited a large sample of children with SSD between the ages of 4 and 7 in which SLI status was free to vary. The children received an extensive speech-language battery and their parents and teachers were asked to fill out DSM-IV ADHD rating scales.

We predicted that ADHD symptom severity would be higher in children meeting criteria for both SSD and SLI compared to the children with SSD and no SLI, consistent with the findings of Beitchman et al. (1989a). Second, we were interested in another subgrouping of children with SSD, namely those with persistent vs. normalized speech problems. Research has shown that $75 \%$ of preschool-aged children with SSD normalize by the time they reach age 6 (Shriberg 1994). We were interested in how the persistent vs. normalized SSD distinction would be associated with the prevalence of ADHD symptomatology in the sample. We predicted that those children with persistent-SSD would be at increased risk for ADHD because there are indications that children who do not improve in their speech-language skills have an increased risk of developing a psychiatric disorder (Baker and Cantwell 1987; Snowling et al. 2006). In summary, the aim of the current study was to investigate the overlap of ADHD and SSD and to consider speech- language subgroups within the SSD sample that may be meaningful for the overlap with ADHD.

\section{Method}

\section{Participants}

Two groups of children between the ages of 4 and 7 years were recruited for this study: children with a history of SSD (SSD: $n=108)$ and children with no history of speech or language difficulties (Controls: $n=41$ ). More SSD children were recruited than controls because the SSD children were part of a genetic linkage study (Smith et al. 2005).

Children with a history of SSD were recruited from public and private schools in metropolitan Denver and through newspaper and radio advertisements. Initially, a letter was sent to all parents of kindergartners in participating school districts indicating that we were seeking children who had a history of articulation difficulties and were (or had been) in speech therapy. Some school districts would not send out a mass mailing to all kindergarteners, but were willing to send a letter to families whose children were already enrolled in speech therapy. As the number of participants that were identified via this school recruitment process did not yield the required sample size, newspaper and radio announcements were used in the local media using the same recruitment language as the letter to parents.

All interested parents of children with potential SSD were given a brief 10-15 min phone screen. The phone screen was intended to (a) ensure that the child had current or previous SSD based on speech therapy history, previous articulation test scores, and/or intelligibility ratings, (b) rule out exclusionary medical conditions: known genetic disorders or syndromes, mental retardation (nonverbal IQ $<70$ ), pervasive developmental disorders (e.g., autism or Asperger's syndrome), significant birth complications, acquired brain injuries, known neurological conditions, peripheral hearing deficits, or peripheral speech anomalies (e.g., cleft palate), and (c) ensure that the child resided in a monolingual English home. Children exposed to foreign languages were excluded, but not children of different ethnic or racial groups whose sole linguistic exposure had been English.

Once children were identified as likely candidates for participation, they came in to our laboratory for testing and were administered the Sounds-in-Words subtest of the Goldman Fristoe Test of Articulation (GF) (Goldman and Fristoe 1986). Further description of the GF subtest is provided below. Children who did not have a history of speech therapy were required to score below the 30th percentile on the GF. In the final sample, 78 children had a history of speech therapy and a GF score at or below the 
30th percentile, 20 children had a history of speech therapy only, and 10 children had a GF score at or below the 30th percentile only.

Control participants were recruited to be similar in age, gender, ethnicity, and socioeconomic status to the SSD probands. They were also required to reside in monolingual English homes. As a first gate to participation, control participants were required to have no history of (a) speech or language difficulties, (b) speech-language therapy, or (c) any of the exclusionary medical conditions listed above. As a second gate to inclusion in the study, control participants must have (a) received a score above the 30th percentile on the GF, and (b) made only developmentally appropriate articulation errors.

The final sample ranged in age from 4 years, 8 months7 years, 4 months with most of the children (71\%) falling in the 5 years, 0 months -6 years, 0 months range. The SSD group was recruited without regard to ADHD or SLI status, which was free to vary. Although the control children could not have a history of speech and/or language difficulties, they were free to vary with respect to ADHD status.

\section{Measures and Procedures}

The Institutional Review Board at the University of Denver approved the study protocol. At the beginning of the study, the examiners described the study procedures to the parents and children. Parents signed a consent form and children over the age of 8 years signed an assent form. The children were tested on a battery of speech, language, and pre- literacy measures over the course of three 2-h sessions. Table 1 lists the measures relevant to the current study. Descriptions of these measures have been previously published by Raitano et al. (2004). Parents and teachers completed questionnaires, including a DSM-IV ADHD rating scale. Parents gave permission for their child's teacher to be mailed the questionnaires. Because teachers were contacted via mailings, there was more missing data for teacher raters than parent raters. Parents, teachers, and children were compensated for their participation.

\section{Articulation Screening}

GF Sounds-in-Words Subtest (Goldman and Fristoe 1986). The Sounds-in-Words subtest is a widely used test of speech sound acquisition that is based on well-developed normative data. The test assesses a child's spontaneous production of speech sounds in single words by asking them to name familiar pictures. The target words were carefully chosen to include all of the single consonant sounds in English (except $/ \mathrm{zh} /$ because of its infrequency) in all of the word positions in which the sounds are commonly found. Common consonant blends are also included.

\section{Speech and Language Subtyping}

Two important subtype distinctions in this sample of SSD probands have previously been identified: persistent or normalized SSD, and presence or absence of comorbid SLI

Table 1 Measures from the speech, language, and cognitive battery

\begin{tabular}{|c|c|c|}
\hline Construct/measure & Reliability & Reference \\
\hline \multicolumn{3}{|l|}{ Articulation } \\
\hline GF-Sounds-in-Words subtest & $0.95^{\mathrm{a}}$ & Goldman and Fristoe 1986 \\
\hline SDCS - Conversational Speech Sample & $0.70^{\mathrm{b}}$ & Shriberg et al. $1997 \mathrm{a}, \mathrm{b}$ \\
\hline \multicolumn{3}{|l|}{ Semantics/syntax } \\
\hline TOLD-P:3-Picture Vocabulary subtest & $0.87^{\mathrm{a}} / 0.81^{\mathrm{c}}$ & Newcomer and Hammill 1997 \\
\hline TOLD — Oral Vocabulary subtest & $0.84^{\mathrm{a}} / 0.86^{\mathrm{c}}$ & Newcomer and Hammill 1997 \\
\hline TOLD — Grammatic Understanding subtest & $0.81^{\mathrm{a}} / 0.80^{\mathrm{c}}$ & Newcomer and Hammill 1997 \\
\hline TOLD — Grammatic Completion subtest & $0.84^{\mathrm{a}} / 0.91^{\mathrm{c}}$ & Newcomer and Hammill 1997 \\
\hline \multicolumn{3}{|l|}{ Nonverbal IQ } \\
\hline DAS_-Pattern Construction subtest & $0.76^{\mathrm{a}} / 0.91^{\mathrm{c}}$ & Elliott 1990 \\
\hline DAS-Matrices subtest & $0.65^{\mathrm{a}} / 0.82^{\mathrm{c}}$ & Elliott 1990 \\
\hline \multicolumn{3}{|l|}{ ADHD } \\
\hline ADHDRS - Inattentive symptoms ${ }^{\mathrm{d}}$ & Parent $0.87^{\mathrm{c}}$, teacher $0.94^{\mathrm{c}}$ & Barkley and Murphy 1998; DuPaul et al. 1998 \\
\hline ADHDRS-Hyperactive/Impulsive symptoms ${ }^{\mathrm{d}}$ & Parent $0.86^{\mathrm{c}}$, teacher $0.95^{\mathrm{c}}$ & Barkley and Murphy 1998; DuPaul et al. 1998 \\
\hline ADHDRS-Impairment ratings ${ }^{\mathrm{d}}$ & Parent $0.94^{\mathrm{c}}$, teacher $0.94^{\mathrm{c}}$ & Barkley and Murphy 1998 \\
\hline
\end{tabular}

DAS $=$ Differential Ability Scales; ADHDRS $=$ DSM-IV ADHD Rating Scale

${ }^{\text {a }}$ Test-retest reliability

${ }^{\mathrm{b}}$ Inter-rater reliability

${ }^{\mathrm{c}}$ Internal consistency reliability

${ }^{\mathrm{d}}$ Reliability calculated in the current study sample 
(Raitano et al. 2004). In this sample, $21 \%$ of the SSD children met criteria for SLI $(n=23)$ and $37 \%$ met criteria for persistent-SSD $(n=39)$, defined as follows.

Comorbid SLI SLI diagnoses were assigned using a modified version of the EpiSLI system described by Tomblin et al. (1996). Four Test of Language Development (TOLD) composites were used to assign an SLI diagnosis: Expressive Language, Receptive Language, Semantics, and Syntax (Newcomer and Hammill 1997). Children were classified as having SLI if they received a standard score below 81 (1.25 SD below the mean) on one or more composites of the TOLD. These four composites are a subset of the standard composites of the TOLD and follow the recommendations for the calculation of the standard composites as recommended by the TOLD manual with minor modifications.

Persistence of Speech Difficulties SSD probands were identified as having persistent or normalized SSD based on a conversational speech sample. A natural conversation between the examiner and the child was recorded using a Sony TCM-5000EV cassette recorder and matching external microphone following recording and on-line glossing procedures described in Shriberg (1993). During this conversation, the child was required to produce at least 50 utterances. Examiners asked questions about the child's interests and hobbies (provided by the parents) until the 50 requisite utterances were obtained. The speech samples were narrowly transcribed using a diacritic system and computer formatting procedures developed specifically for research on child speech disorders (Shriberg et al. 2001). The speech samples were used as input to a computerized version of the Speech Disorders Classification System (SDCS: Shriberg et al. 1997a, b), a classification instrument used to categorize children (and adults) with speech disorders based on a large corpus of lifespan data of individuals with disordered and typical speech acquisition. The SDCS has been used extensively in speech-genetics studies (e.g., Shriberg et al. 2006; Smith et al. 2005) and in two large epidemiological studies (Campbell et al. 2003; Shriberg et al. 1999).

For the purposes of the present study, the two disordered SDCS classifications, termed Speech Delay (SD) and Normalized Speech Acquisition/Speech Delay (which is intermediate between SD and Normalized Speech Acquisition), were combined to form the category of persistentSSD. In this classification scheme, children with persistent age-inappropriate speech sound omissions and/or substitutions are classified as having persistent-SSD. In contrast, children with no omissions/substitutions or with only ageappropriate speech sound distortions were classified as having Normalized Speech Acquisition in the SDCS system. We considered these children to have normalized$\mathrm{SSD}$, or in the case of controls, to have normal speech acquisition. Two children in the SSD sample had missing data for the SDCS classification.

\section{$A D H D$}

Symptom Ratings Parents and teachers completed a DSMIV ADHD Rating Scale for each child similar to other DSM-IV ADHD questionnaires (Barkley and Murphy 1998; DuPaul et al. 1998). Each DSM-IV ADHD symptom was rated on a four-point scale $(0=$ not at all, $1=$ just a little, $2=$ pretty much/quite a bit ${ }^{1}, 3=$ very much). The parent and teacher questionnaires contained the same 18 items. Questionnaires similar to the one used in this study have been shown to be reliable and valid in samples with a similar age range to this study (DuPaul et al. 1997, 1998). In this sample, parent and teacher ratings correlated at $r(104)=0.30$ for inattentive symptoms and $r(104)=0.32$ for hyperactive/impulsive symptoms, consistent with previous studies (Mitsis et al. 2000).

Questionnaires in which raters skipped three or more items were not included in the analyses, as recommended by DuPaul et al. (1998). One parent and three teachers omitted three or more items and their ratings were not considered. Forty-two subjects (28\%, 21 SSD-only, 5 SSD + SLI, and 16 controls) were excluded from the teacher questionnaire analysis because the teacher did not return the questionnaire to the study. The excluded children did not differ significantly from the children that remained in the analysis on any of the demographic variables listed in Table 2. However, there was a trend for teachers to return more questionnaires for children in the SSD group than the control group, $\chi^{2}(1, n=149)=$ $3.40, p=0.065$. Based on this trend, we were concerned that teachers may have had a bias to return questionnaires for children with higher ADHD symptoms. We compared children whose teacher did and did not return the questionnaire on their parent's ratings of their inattentive and hyperactive symptoms. Results showed no evidence for a bias in the inattentive domain, $t(73.4)^{2}=1.2, n s$, but a trend for teachers to return questionnaires for children with less severe hyperactive/impulsive symptoms, $t(70.1)=1.85$, $p=0.068$.

\footnotetext{
${ }^{1}$ The anchor for a rating of 2 on the parent questionnaire was "pretty much" whereas the anchor for a rating of 2 on the teacher questionnaire was "quite a bit."

${ }^{2}$ Degrees of freedom that are decimals are from t-tests in which equality of variances was not assumed. In all cases, if the significance value when equality of variances was assumed versus not assumed differed, the corrected t-test was reported.
} 
Table 2 Demographics for SSD and control sample broken down by SLI status

\begin{tabular}{|c|c|c|c|c|}
\hline Demographics & $\begin{array}{l}\mathrm{SSD}+\mathrm{SLI}(n=23) \\
\mathrm{M}(\mathrm{SD})\end{array}$ & $\begin{array}{l}\text { SSD-only }(n=85) \\
\text { M (SD) }\end{array}$ & $\begin{array}{l}\text { Controls }(n=41) \\
M(S D)\end{array}$ & Significance test \\
\hline Age in months & $68.9(8.3)$ & $68.7(8.1)$ & $67.5(5.2)$ & $F(2,146)<1, n s$ \\
\hline Nonverbal $\mathrm{IQ}^{\mathrm{a}}$ & $94.8(12.1)$ & $106.3(10.3)$ & $111.6(8.5)$ & $F(2,145)=20.32, p<0.001$ \\
\hline Gender & $61 \%$ Males & $58 \%$ Males & $71 \%$ Males & $\chi^{2}(2, n=149)=2.01, n s$ \\
\hline Ethnicity & $70 \%$ Caucasian & $82 \%$ Caucasian & $85 \%$ Caucasian & $\chi^{2}(2, n=148)=2.54, n s$ \\
\hline Mother education (years) & $15.0(1.8)$ & $15.8(2.5)$ & $16.2(2.8)$ & $F(2,145)=1.81, n s$ \\
\hline Father education (years) & $15.2(2.3)$ & $15.6(2.4)$ & $16.0(2.5)$ & $F(2,143)<1, n s$ \\
\hline
\end{tabular}

${ }^{a}$ For nonverbal IQ, Tukey HSD post-hoc tests showed SSD + SLI $<$ SSD-only $<$ Controls

Both categorical and continuous data analytic approaches were used to analyze the ratings from the DSM-IV ADHD rating scale. When discussing the categorical results, we will refer to children who meet the DSM-IV symptom criteria for ADHD as having ADHD although we recognize that this methodology is not DSM-IV compliant because it does not take into account functional impairment, duration of symptoms, age of onset, or the number of contexts in which the symptoms occur. Nevertheless, we will use this nomenclature for the purposes of simplicity and brevity, although it is important to point out the caveat that children in our ADHD groups only meet the symptom criteria for ADHD and may not meet formal DSM-IV clinical criteria.

For the categorical ADHD analyses, we used the Or rule as a strategy for combining information from two raters. This strategy was used in the DSM-IV field trials (Lahey et al. 1994) and is considered optimal for identifying children with impairing ADHD, including children in the age range of this study (Lahey et al. 1998). The Or rule stipulates that a symptom should be considered present if it is endorsed at a clinically significant level by a parent or a teacher (e.g., a rating of 2 or 3). We used the DSM-IV diagnostic cut-offs of 6 or more symptoms in one or both domains to assign the symptom-level diagnosis of inattentive, hyperactive-impulsive, or combined type ADHD.

A continuous approach to data analysis with the ADHD questionnaire data was also employed. Because each symptom was rated by parents and teachers on a scale of 0 to 3 , we averaged the ratings within the inattentive and hyperactive-impulsive domains. It is important to point out that taking the mean of ordinal data makes the interpretation of the results specific to the $0-3$ scaling employed by this questionnaire, because the data are not interval.

Impairment To address one limitation noted above, that the Or rule categorical designations do not take into account impairment associated with ADHD symptoms, a crucial dimension for diagnosis in the DSM-IV, we also analyzed the impairment questions that were part of the DSM-IV ADHD rating scale. The impairment questions were modeled after those used by Barkley and Murphy (1998). For these items, parents and teachers were asked to rate the extent to which the ADHD symptoms they endorsed interfered with the child's ability to function in different contexts. The rating scale was a four-point scale $(0=$ not at all, 1=sometimes, 2=often, $3=$ very often). A missing data criterion was set such that one item could be skipped, but not two or more. Three parent ratings and six teacher ratings were not analyzed because they did not meet this criterion.

\section{Analyses}

We employed an error rate of $p<0.05$ but we noted trends of $p<0.1$, particularly when there was convergence between the parent and teacher ratings. For the continuous analyses, we chose to use ANCOVAs, covarying nonverbal IQ because of group differences in this sample and the potential negative relationship between nonverbal IQ and ADHD symptoms (APA 2000; Werry et al. 1987). We did not covary age in these analyses because the age range was small in this study. The homogeneity of regression assumption was checked for all of the covariates according to recommendations by Tabachnick and Fidell (2001) and found to be satisfactory for all analyses. Because the $n$ 's differed considerably in many of the analyses, we also examined the equality of variance assumption. We considered this assumption to be violated if the ratio of the largest to smallest variance exceeded 3:1 (Tabachnick and Fidell 2001). In most cases, the assumption was not violated. For the few cases in which the assumption was violated, we judged that the bias would be in the conservative direction because the largest group had the largest variance or that there would be no systematic bias because the largest variances occurred in the smallest and largest groups. 
Table 3 Demographics for SSD sample broken down by persistence status and SLI status

\begin{tabular}{|c|c|c|c|c|c|}
\hline Demographics & $\begin{array}{l}\text { Persistent-SSD + } \\
\text { SLI }(n=9) \\
\text { M (SD) }\end{array}$ & $\begin{array}{l}\text { Persistent-SSD, no } \\
\text { SLI }(n=30) \\
\text { M (SD) }\end{array}$ & $\begin{array}{l}\text { Normalized-SSD + } \\
\text { SLI }(n=13) \\
\text { M (SD) }\end{array}$ & $\begin{array}{l}\text { Normalized-SSD, } \\
\text { no SLI }(n=54) \\
\text { M (SD) }\end{array}$ & $\begin{array}{l}\text { Significance } \\
\text { Test }\end{array}$ \\
\hline Age in months & $65.7(7.5)$ & $66.2(6.7)$ & $71.3(8.7)$ & $70.1(8.6)$ & $F(3,102)=2.42, p=0.07$ \\
\hline Nonverbal $\mathrm{IQ}^{\mathrm{a}}$ & $96.9(12.1)$ & $105.8(11.2)$ & $92.6(12.5)$ & $106.9(9.7)$ & $F(3,101)=7.81, p<0.001$ \\
\hline Gender & $56 \%$ Males & $67 \%$ Males & $69 \%$ Males & $54 \%$ Males & $\chi^{2}(3, n=106)=1.96, n s$ \\
\hline Ethnicity & $89 \%$ Caucasian & $77 \%$ Caucasian & $54 \%$ Caucasian & $85 \%$ Caucasian & $\chi^{2}(3, n=105)=6.71, p=0.08$ \\
\hline Mother education (years) & $14.0(1.2)$ & $15.3(2.7)$ & $15.9(1.7)$ & $16.1(2.4)$ & $F(3,101)=2.47, p=0.07$ \\
\hline Father education (years) & $14.8(2.1)$ & $15.0(2.2)$ & $15.7(2.3)$ & $16.0(2.5)$ & $F(3,99)=1.39, n s$ \\
\hline
\end{tabular}

${ }^{a}$ For Nonverbal IQ, Tukey HSD post-hoc tests showed Normalized-SSD + SLI $=$ Persistent-SSD + SLI $<$ Persistent-SSD, no SLI $=$ NormalizedSSD, no SLI

\section{Results}

\section{Sample Demographics}

Table 2 summarizes the demographic information for children in the SSD + SLI, SSD-only, and control groups. Table 3 further decomposes the SSD sample by adding a second categorizing dimension, persistence of SSD, in addition to SLI status. In both tables, the groups only differed significantly on nonverbal IQ, which was covaried in all subsequent analyses.

ADHD Symptomatology in Children with SSD + SLI, SSD-only, and Controls

For the categorical analyses, we assigned three symptom-level ADHD subtypes consistent with the DSM-IV categories: Inattentive (ADHD-I), Hyperactive-Impulsive (ADHD-HI), and Combined type (ADHD-C). We also constructed a summary variable, ADHD-any, which included all children meeting criteria for any ADHD subtype. These categories only considered the level of ADHD symptomatology, not the additional DSM-IV criteria that are necessary for a formal clinical diagnosis of ADHD. Chi-Square analyses were conducted comparing rates of ADHD in the SSD-only sample and the SSD + SLI sample to the control sample. The Fisher's Exact statistic was also used because the expected count in the cell for controls meeting ADHD criteria was often less than 5 . In the SSD-only sample, there was not an increased rate of
ADHD compared to controls for ADHD-any or any of the ADHD subtypes (see Table 4). When the parent and teacher raters were analyzed separately, the same results were obtained.

In contrast, in the SSD + SLI sample, there was an increased rate of ADHD-any compared to controls, $\chi^{2}(1$, $n=64)=15.05, p<0.001$, Fisher's Exact Test $p<0.001$, that was driven primarily by increased rates of ADHD-I, $\chi^{2}(1$, $n=64)=14.01, p<0.001$. When parents and teacher raters were analyzed separately, results showed that teacher ratings were primarily driving these findings. According to teachers, there was an increased rate of ADHD-any, $\chi^{2}$ $(1, n=41)=9.92, p<0.01$, Fisher's Exact Test $p<0.01$, that was driven primarily by increased rates of ADHD-I, $\chi^{2}(1$, $n=41)=8.04, p<0.01$, Fisher's Exact Test $p<0.01$. Parent raters only showed a trend toward an increase in ADHDany rates in children with SSD + SLI, $\chi^{2}(1, n=63)=3.59$, $p=0.06$, Fisher's Exact Test $p=0.13$. In summary, the SSDonly group did not show an increased rate of ADHD compared to controls whereas the SSD + SLI group showed higher rates of ADHD-I. The teacher ratings primarily drove these significant findings, although the parent ratings were in the same direction.

We also used the ADHD rating scale as a continuous measure to calculate mean symptom severity ratings for the inattentive and hyperactive-impulsive clusters of symptoms. Group comparisons were conducted using one-way ANCOVAs with group as the independent variable (3 levels: SSD + SLI, SSD-only, control) and nonverbal IQ

Table 4 Or rule ratings of ADHD-I, ADHD-HI, ADHD-C and ADHD-any

\begin{tabular}{lllll}
\hline & ADHD-I & ADHD-HI & ADHD-C & ADHD-any \\
\hline SSD + SLI $(n=23)$ & $7(30 \%)^{\mathrm{a}}$ & $0(0 \%)$ & $2(9 \%)$ & $9(39 \%)^{\mathrm{a}}$ \\
SSD-only $(n=85)$ & $4(5 \%)$ & $4(5 \%)$ & $3(4 \%)$ & $11(13 \%)$ \\
Control $(n=41)$ & $0(0 \%)$ & $1(2 \%)$ & $0(0 \%)$ & $1(2 \%)$ \\
\hline
\end{tabular}

${ }^{\text {a }}$ Rates significantly higher than controls, $p<0.001$ 
covaried. The main effect of group was nonsignificant in the parent inattention severity ratings, $F(2,143)=0.95$, $n s$, partial $\eta^{2}=0.01$ parent hyperactive-impulsive severity ratings, $F(2,143)=0.47, n s$, partial $\eta^{2}=0.01$ and the teacher hyperactive-impulsive ratings, $F(2,100)=1.37, n s$, partial $\eta^{2}=0.03$. Similar to the Chi-square analyses above, only the teacher inattention ratings showed a significant main effect of group, $F(2,100)=7.8, p=0.001$, partial $\eta^{2}=0.14$. Tukey HSD post-hoc tests showed that the main effect of group was driven by a significant increase in ADHD inattentive ratings for the SSD + SLI group as compared to the SSDonly group, $p<0.001$, and the control group, $p<0.001$. The SSD-only and control groups did not differ significantly from each other. Figure 2 shows a plot of parent and teacher mean severity ratings of inattentive and hyperactiveimpulsive symptoms.

In order to determine if the increased symptom severity in the SSD + SLI group was associated with impairment in daily functioning, we conducted one-way ANCOVAs with group as the independent variable (3 levels: SSD + SLI, SSD-only, control) and nonverbal IQ covaried. The dependent variable for this analysis was the average impairment ratings. The results showed a non-significant trend for the parent impairment ratings to differ across groups, $F(2,140)=2.37, p<0.1$, partial $\eta^{2}=0.03$. This analysis reached significance for the teacher impairment ratings, $F(2,94)=6.10, p<0.01$, partial $\eta^{2}=0.12$. Tukey HSD post-hoc tests showed that the main effect of group was driven by a significant increase in teacher impairment ratings for the SSD + SLI group $(M=1.21, S D=0.68)$ as compared to the SSD-only group $(M=0.60, S D=0.78), p<$ 0.01 , and the control group $(M=0.17, S D=0.34), p<0.001$. In this analysis, the SSD-only group also showed increased impairment ratings compared to the controls, $p<0.05$. This last comparison was the only evidence across several analyses that the SSD-only group showed an increase in ADHD symptomatology compared to the controls.

In summary, the analyses of ADHD symptomatology (both categorical and continuous) and impairment showed that the SSD + SLI group had increased ADHD symptomatology, specifically symptoms of inattention, compared to the SSD-only group and controls. In general, the SSD-only group did not show increased ADHD symptomatology compared to controls. Teacher ratings of inattention tended to show stronger effects although parent ratings tended to show the same trends.

\section{Speech-Language Subgroup Analyses}

We conducted 2 (SLI vs. no SLI) $\times 2$ (persistent-SSD vs. normalized-SSD) ANCOVAs, controlling for the effects of nonverbal IQ on parent and teacher mean severity ratings. For the parent and teacher hyperactive-impulsive mean severity ratings, there were no significant main effects of persistence or SLI status, nor was there a significant interaction between persistence and SLI. However, for the parent inattention mean severity ratings, there was a significant main effect of persistence $F(1,100)=9.87$, $p<0.01, \eta^{2}=0.09$, which was qualified by a trend towards a persistence x SLI interaction, $F(1,100)=3.11, p=0.08$, $\eta^{2}=0.03$. Post-hoc t-tests revealed that the interaction was due to the fact that SLI status tended to be associated with an increase in inattention mean severity ratings when articulation difficulties had normalized, $t(15.8)=2.02, p=$ 0.06 , but not if the articulation problems persisted, $t(37)=$ 0.41 , ns. The teacher inattention ratings yielded similar results; there was a main effect of persistence, $F(1,74)=$ 8.91, $p<0.01, \eta^{2}=0.11$, as well as a main effect of SLI status, $F(1,74)=12.24, p<0.01, \eta^{2}=0.14$. As with the parent ratings, these main effects were qualified by a significant persistence $\times$ SLI interaction, $F(1,74)=7.44, p<$ $0.01, \eta^{2}=0.09$. Once again, post-hoc t-tests revealed that SLI status was associated with an increase in inattention mean severity ratings when articulation difficulties had normalized, $t(49)=5.68, p<0.001$, but not if the articulation problems persisted, $t(26)=1.13, n s$. The graphs in Fig. 3 illustrate this interaction. In summary, the ANCOVAs revealed that a particular group of children, those with
Fig. 2 Parent and teacher mean severity ratings of inattentive and hyperactive-impulsive symptoms. Error bars reflect the standard error of the mean
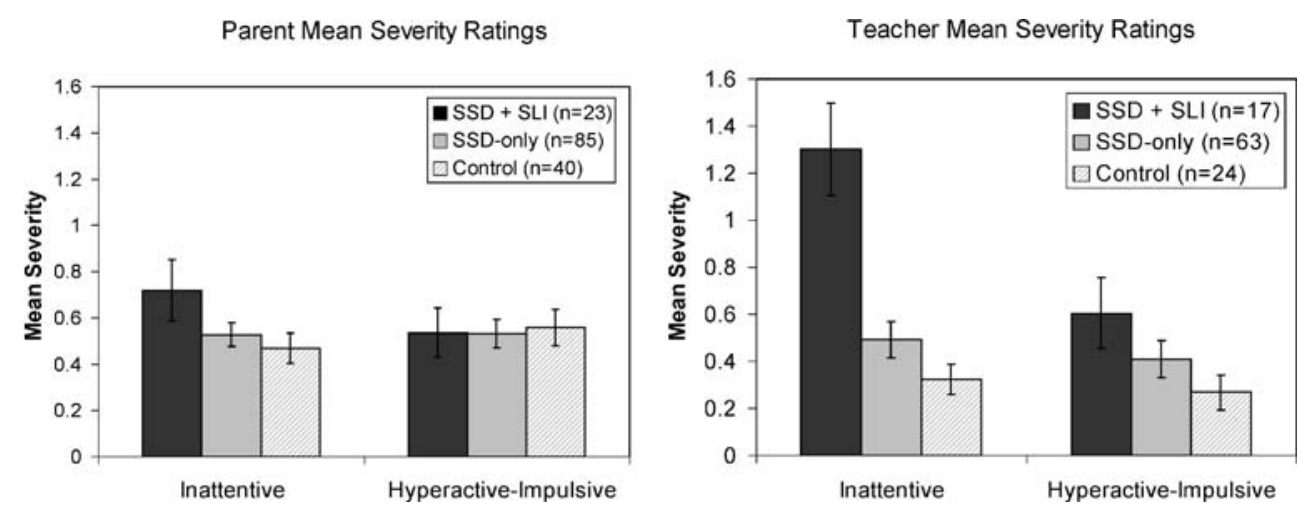
Parent Inattention Mean Severity Ratings

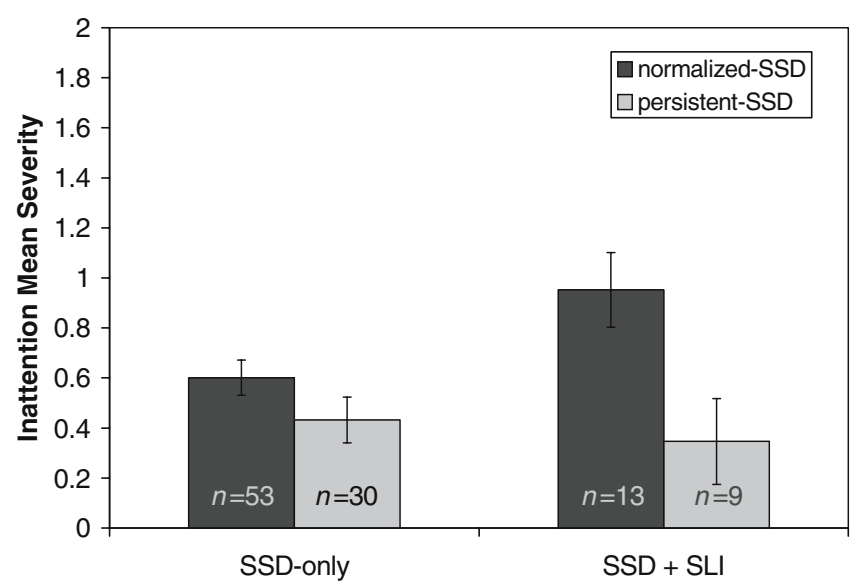

Teacher Inattention Mean Severity Ratings

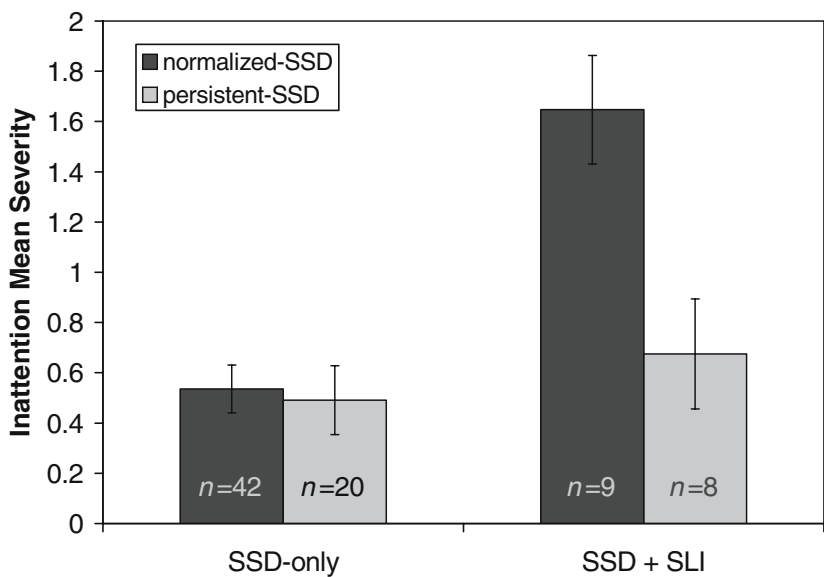

Fig. 3 Results of 2 (SLI vs. non-SLI) $\times 2$ (persistent-SSD vs. normalized-SSD) ANCOVAs on parent and teacher inattention mean severity ratings. Means are adjusted for the covariates and error bars reflect the standard error of the mean

SLI and normalized-SSD, tended to have the highest inattention mean severity ratings, a finding that ran counter to one of our hypotheses.

We conducted follow-up 2 (SLI vs. not-SLI) $\times 2$ (persistent-SSD vs. normalized-SSD) ANOVAs on several demographic variables to determine if any of these variables showed the same SLI x persistence interaction which could help to explain the unpredicted finding. Age, nonverbal IQ, and mother's and father's years of education were used as dependent variables. None of these dependent variables showed an SLI $\times$ persistence interaction $(\mathrm{SLI} \times$ persistence for age: $F(1,102)<1, n s$, $\eta^{2}=0.002$; SLI $\times$ persistence for nonverbal IQ: $F(1,101)=$ 1.08, $p=0.30, \eta^{2}=0.01$; SLI x persistence for mother's education: $F(1,101)<1, n s, \eta^{2}=0.01$; SLI x persistence for father's education: $\left.F(1,99)<1, n s, \eta^{2}=0.000\right)$. Additionally, a Chi-square analysis of gender and ethnicity did not reveal any significant differences across the speech-language subgroups (see Table 3). Thus, none of the demographic variables showed the same SLI $\times$ persistence interaction found in the ADHD inattentive ratings.

All of the children that met Or rule criteria for ADHD in the SSD + SLI sample belonged to the SLI + normalizedSSD group. Additionally, 9 of the 13 children (69\%) in the SLI + normalized-SSD group met $\mathrm{Or}$ rule criteria for ADHD. Removing these children from the earlier categorical and continuous analyses revealed that the group differences between the SSD + SLI group and controls were primarily driven by this subgroup of children. For the analyses of mean severity ratings, when the $13 \mathrm{SLI}+$ normalized-SSD children were removed from teacher inattention analysis, the previously significant main effect of group disappeared, $F(2$, $91)=0.47, n s$, with the effect size diminishing from partial $\eta^{2}=0.14$ to partial $\eta^{2}=0.01$.
In summary, the subgroup analyses revealed a distinct group of 13 children with SLI and normalized-SSD that were primarily responsible for the finding that the SSD + SLI group showed increased rates of ADHD. The fact that these children with normalized articulation deficits and SLI were rated worse on ADHD inattentive symptoms than SLI children with persistent articulation deficits is a finding that ran counter to one of our hypotheses. Demographic variables could not explain the unexpected directionality. Possible explanations for this finding will be explored in the discussion.

\section{Discussion}

The overall goal of this study was to investigate the overlap of ADHD and SSD because this comorbidity has been less well-studied than other comorbidities associated with these disorders. Our first hypothesis was that the SSD + SLI group would show increased rates of ADHD symptomatology compared to the SSD-only and control group. Consistent with our hypothesis, results showed that the SSD + SLI group showed higher rates of ADHD (specifically ADHD-I type), higher symptom severity ratings for the inattentive domain, and higher impairment ratings compared to the SSD-only group and controls, replicating the results of Beitchman et al. (1989a). The findings were primarily driven by the teacher ratings although the parent ratings were always in the same direction. In contrast to these significant group differences, the SSD-only group did not show converging evidence for increased rates of ADHD symptomatology compared to controls.

The second analysis examined another subgrouping within the SSD sample, namely those with persistent vs. normalized SSD. We hypothesized that children with 
persistent-SSD would be at increased risk for ADHD because previous research had indicated that persistent speech-language problems tended to be associated with a higher risk of developing a psychiatric disorder (Baker and Cantwell 1987; Snowling et al. 2006). We were also interested in whether the persistence variable would interact with the comorbid SLI variable to predict ADHD symptomatology. Results showed an interaction of the SLI and persistence variables that drove all other main effects. Interestingly, the interaction was in the opposite direction from one of our hypotheses, such that children with SLI + normalized-SSD had more severe ADHD inattentive symptoms than any other group. A discussion of this subgroup of children will follow a more general discussion of patterns in the results.

Two patterns were evident from the results of our group comparisons and speech-language subgroup analyses. First, the findings were specific to the inattentive cluster of symptoms. This finding mirrors research investigating the overlap of RD and ADHD which tends to find a stronger connection between reading and the inattentive symptoms of ADHD than the hyperactive/impulsive symptoms (Lahey et al. 1998; Willcutt and Pennington 2000). Together with our findings, these results suggest that the inattentive symptoms of ADHD might be more strongly associated with reading and language difficulties than the hyperactive/ impulsive symptoms.

Secondly, the results were always stronger for teacher ratings, although the parent ratings were in the same direction. The possibility that this finding may have resulted from bias introduced by missing teacher data is discussed further below. However, our analyses suggest that any bias introduced from missing teacher data was likely to be in the conservative direction. An alternative explanation for the stronger effects in the teacher data is that teachers make more attentional demands than parents of children in the age range we were studying, primarily kindergartners and first-graders. Thus, teachers may have more of an opportunity to observe a child's attentional limitations compared to his/her peers. Although this hypothesis is merely speculative, there is evidence that teachers are perceived as better raters of inattention/hyperactivity by mental health professionals (Loeber et al. 1990) and parents (Phares 1997).

We now turn to the speech-language subgroup analysis and the unexpected finding that the children with SLI and normalized-SSD were driving the finding that the SSD + SLI group had higher ADHD symptomatology than the SSD-only and control group. Obviously, our subgroups of SLI + persistent-SSD and SLI + normalized-SSD children were small, which leaves open the possibility that this finding would not generalize to the population. However, we found the same interaction effect in teacher and parent ratings (albeit only a trend in the parent ratings), which lends some support to the stability of the findings.

One explanation for these findings is that the SSD-only group and the SLI + persistent-SSD group may possess a circumscribed phonological deficit that places them at risk for speech and language problems, but not ADHD. In these children, their phonological deficit may affect their speech and perhaps their language, but because the deficit is primarily limited to phonological processing, it does not affect attention directly. In contrast, children with SLI + normalized-SSD may possess a more general cognitive deficit that delays their speech acquisition and places them at risk for language and attention difficulties. These children may have difficulty acquiring any new skill, including speech, language, and attention, but the nature of their speech problem is transient because it can be attributed to a more general cognitive delay or "neurodevelopmental immaturity" (Beitchman and Inglis 1991, p. 107). Although this hypothesis is merely speculative, it does derive some support from a recently published study using substantively the same sample of children as this study. In this prior study, Raitano et al. (2004) found that the SLI + normalized-SSD children were significantly worse than the other subgroups on a measure of processing speed and there was a trend for these children to have significantly lower nonverbal IQ scores. Although the nonverbal IQ trend did not hold up in the present sample, which included several children who were outside the age range of the Raitano et al. study, the mean nonverbal IQ pattern from this study was supportive of the neurodevelopmental immaturity hypothesis as the SLI + normalized-SSD children had the lowest mean nonverbal IQ. Taken together, the results of the Raitano et al. (2004) study and the current study highlight the SLI + normalized-SSD subset of children as differentially impaired on several cognitive and attention measures. Future research will be needed to determine the exact nature of these children's deficits.

The primary limitations of this study are the possible bias resulting from missing teacher data and the small sample size in some of the speech-language subgroups. As noted, $28 \%$ of the teachers did not return the ADHD questionnaire to the study. We attempted to determine if there was any systematic bias to return the questionnaire that was a function of the child's ADHD behaviors. There was no detectable bias for the inattentive symptoms, the domain in which the main findings of the study were obtained. There was a trend for teachers to return questionnaires for children with less severe hyperactive/ impulsive symptoms. This result may have been indicative of a general reluctance on the part of teachers to return questionnaires that would rate children in the impaired direction. In this case, our findings would not be an artifact of missing data. In fact, this result suggests that our 
findings would have been stronger if the missing data was returned. However, it remains a possibility that there was a bias in the opposite direction that could not be detected. Importantly, there was good convergence between the parent and teacher reports, although the parent report did not always reach statistical significance.

The second limitation of the study was the small sample sizes within some speech-language subgroups that may limit the generalizability of the findings. Additionally, the limited power restricted us from investigating potentially interesting moderators, such as gender. ADHD shows a strong gender bias ranging from 3:1-9:1 (boy/girls) (Willcutt and Pennington 2000). An interesting future direction would be to explore whether males with SSD and SLI are differentially at risk for ADHD compared to females. In this study, we were merely able to establish that our speech-language subgroups did not have significantly different gender ratios which could account for the findings.

Overall, the practical implications of these findings are that children with comorbid SSD and SLI should be considered at risk for ADHD, whereas children with SSDonly do not seem to be at increased risk for ADHD compared to controls. Although the persistent/normalized status of a child's SSD may be useful in determining ADHD risk, the fact that this judgment is a protracted one limits the clinical utility of this speech status dimension. Both psychiatrists and psychologists treating children with ADHD and speech-language pathologists treating children with SSD and SLI should be aware of these potential comorbidities (Baker and Cantwell 1982; Cantwell et al. 1980; Hill 2000; Love and Thompson 1988).

The etiological explanations for these findings remain somewhat of a puzzle. Turning back to the Venn diagrams in Fig. 1, it appears that the left figure is appropriate if the population sampled is diagnosed with SSD-only. However, the right figure more accurately summarizes the data if the population sampled is diagnosed with SSD and SLI. Despite the lack of phenotypic association between SSDonly and ADHD, recent molecular genetic evidence has shown that SSD and ADHD are linked to the same locus on chromosome 6p (Smith et al. 2005; Willcutt et al. 2002). Thus, the phenotypic and genetic levels of analysis are not showing convergence. One explanation that is being investigated in our lab is that the linkage of SSD to $6 p$ could have been driven by a subset of children with SSD and SLI. If this is the case, then it may explain why the SSD-only group did not show increased rates of ADHD, but the SSD + SLI group did. However, a puzzle would still remain because two independent genome-screens of SLI loci have not identified a risk locus on $6 \mathrm{p}$ (Bartlett et al. 2002; SLI Consortium 2002, 2004). Alternatively, SSD and ADHD may share one or more genetic risk loci but the complex multifactorial genetic etiology of both disorders (Pennington 2006) may make these overlapping loci, especially if they have small effect sizes, insufficient to cause detectable phenotypic overlap. Clearly, more research needs to be conducted to dissect the etiological and phenotypic overlap among this cluster of comorbid disorders.

Acknowledgements The present study was supported by the National Institute of Child Health and Human Development (HD049027-22). The authors wish to extend their gratitude to the children and families who participated in this research and to our collaborators who helped collect the data for this project. Special thanks to Erik Willcutt and Nancy Raitano for their help at the inception of this project. We also wish to thank Irina Kaminer, Robin Peterson, and Erin Phinney for their helpful comments on earlier drafts of this manuscript.

\section{References}

American Psychiatric Association (APA). (1980). Diagnostic and statistical manual of mental disorders (3rd ed.). Washington, D.C.: APA.

American Psychiatric Association (APA). (1987). Diagnostic and statistical manual of mental disorders (3rd ed., revised). Washington, D.C.: APA.

American Psychiatric Association (APA). (2000). Diagnostic and statistical manual of mental disorders (4th ed., text revision). Washington, D.C.: APA.

Baker, L., \& Cantwell, D. P. (1982). Developmental, social and behavioral characteristics of speech and language disordered children. Child Psychiatry and Human Development, 12, 195-206.

Baker, L., \& Cantwell, D. P. (1985). Psychiatric and learning disorders in children with speech and language disorders: A critical review. Advances in Learning and Behavioral Disabilities, 4, 1-28.

Baker, L., \& Cantwell, D. P. (1987). A prospective psychiatric followup of children with speech/language disorders. Journal of the American Academy of Child and Adolescent Psychiatry, 26, $546-553$.

Baker, L., \& Cantwell, D. P. (1992). Attention deficit disorder and speech/language disorders. Comprehensive Mental Health Care, 2, 3-16.

Bakker, S. C., van der Meulen, E. M., Buitelaar, J. K., Sandkuijl, L. A., Pauls, D. L., Monsuur, A. J., et al. (2003). A wholegenome scan in 164 Dutch sib pairs with attention-deficit/ hyperactivity disorder: Suggestive evidence for linkage on chromosomes 7p and 15q. American Journal of Human Genetics, $72,1251-1260$.

Barkley, R. A., \& Murphy, K. R. (1998). Attention-deficit hyperactivity disorder: A clinical workbook (2nd ed.). New York: Guilford.

Bartlett, C. W., Flax, J. F., Logue, M. W., Vieland, V. J., Bassett, A. S., Tallal, P., et al. (2002). A major susceptibility locus for specific language impairment is located on 13q21. American Journal of Human Genetics, 71, 45-55.

Beitchman, J. H., Hood, J., \& Inglis, A. (1990). Psychiatric risk in children with speech and language disorders. Journal of Abnormal Child Psychology, 18, 283-296.

Beitchman, J. H., Hood, J., Rochon, J., \& Peterson, M. (1989a). Empirical classification of speech/language impairment in children. II. Behavioral characteristics. Journal of the American Academy of Child and Adolescent Psychiatry, 28, 118-123. 
Beitchman, J. H., Hood, J., Rochon, J., Peterson, M., Mantini, T., \& Majumdar, S. (1989b). Empirical classification of speech/ language impairment in children. I. Identification of speech/ language categories. Journal of the American Academy of Child and Adolescent Psychiatry, 28, 112-117.

Beitchman, J. H., \& Inglis, A. (1991). The continuum of linguistic dysfunction from pervasive developmental disorders to dyslexia. Psychiatric Clinics of North America, 14, 95-111.

Beitchman, J. H., Nair, R., Clegg, M., Ferguson, B., \& Patel, P. G. (1986). Prevalence of psychiatric disorders in children with speech and language disorders. Journal of the American Academy of Child and Adolescent Psychiatry, 25, 528-535.

Bishop, D. V. M. (1997). Uncommon understanding: Development and disorders of language comprehension in children. Cambridge: Psychology Press.

Bishop, D. V. M. (2006). What causes specific language impairment in children? Current Directions in Psychological Science, 15, 217-221.

Bishop, D. V. M., \& Adams, C. (1990). A prospective study of the relationship between specific language impairment, phonological disorders and reading retardation. Journal of Child Psychology and Psychiatry, 31, 1027-1050.

Campbell, T. F., Dollaghan, C. A., Rockette, H. E., Paradise, J. L., Feldman, H. M., Shriberg, L. D., et al. (2003). Risk factors for speech delay of unknown origin in 3-year-old children. Child Development, 74, 346-357.

Cantwell, D. P., \& Baker, L. (1985). Psychiatric and learning disorders in children with speech and language disorders: A descriptive analysis. Advances in Learning and Behavioral Disabilities, 4, 29-47.

Cantwell, D. P., Baker, L., \& Mattison, R. E. (1980). Psychiatric disorders in children with speech and language retardation. Factors associated with development. Archives of General Psychiatry, 37, 423-426.

DuPaul, G. J., Power, T. J., Anastopoulos, A. D., \& Reid, R. (1998). $A D H D$ rating scale-IV: Checklists, norms, and clinical interpretation. New York: Guilford.

DuPaul, G. J., Power, T. J., Anastopoulos, A. D., Reid, R., McGoey, K. E., \& Ikeda, M. J. (1997). Teacher ratings of attention deficit hyperactivity disorder symptoms: Factor structure and normative data. Psychological Assessment, 9, 436-444.

Elliott, C. D. (1990). Differential ability scales. San Antonio: The Psychological Corporation.

Fisher, S. E., \& DeFries, J. C. (2002). Developmental dyslexia: Genetic dissection of a complex cognitive trait. Nature Reviews Neuroscience, 3, 767-780.

Gallagher, A., Frith, U., \& Snowling, M. J. (2000). Precursors of literacy delay among children at genetic risk of dyslexia. Journal of Child Psychology and Psychiatry, 41, 203-213.

Gayan, J., Willcutt, E. G., Fisher, S. E., Francks, C., Cardon, L. R., Olson, R. K., et al. (2005). Bivariate linkage scan for reading disability and attention-deficit/hyperactivity disorder localizes pleiotropic loci. Journal of Child Psychology and Psychiatry, 46, 1045-1056.

Goldman, R., \& Fristoe, M. (1986). The Goldman Fristoe Test of Articulation. Circle Pines, MN: American Guidance Service.

Gualtieri, C. T., Koriath, U., Van Bourgondien, M., \& Saleeby, N. (1983). Language disorders in children referred for psychiatric services. Journal of the American Academy of Child and Adolescent Psychiatry, 22, 165-171.

Hill, G. P. (2000). A role for the speech-language pathologist in multidisciplinary assessment and treatment of attention-deficit/ hyperactivity disorder. Journal of Attention Disorders, 4, 69-79.

Lahey, B. B., Applegate, B., McBurnett, K., Biederman, J., Greenhill, L., Hynd, G. W., et al. (1994). DSM-IV field trials for attention deficit hyperactivity disorder in children and adolescents. American Journal of Psychiatry, 151, 1673-1685.

Lahey, B. B., Pelham, W. E., Stein, M. A., Loney, J., Trapani, C., Nugent, K., et al. (1998). Validity of DSM-IV attention-deficit/ hyperactivity disorder for younger children. Journal of the American Academy of Child and Adolescent Psychiatry, 37, 695-702.

Loeber, R., Green, S. M., \& Lahey, B. B. (1990). Mental health professionals' perception of the utility of children, mothers, and teachers as informants on childhood psychopathology. Journal of Clinical Child Psychology, 19, 136-143.

Loo, S. K., Fisher, S. E., Francks, C., Ogdie, M. N., MacPhie, I. L., Yang, M., et al. (2004). Genome-wide scan of reading ability in affected sibling pairs with attention-deficit/hyperactivity disorder: Unique and shared genetic effects. Molecular Psychiatry, 9, 485493.

Love, A. J., \& Thompson, M. G. (1988). Language disorders and attention deficit disorders in young children referred for psychiatric services: Analysis of prevalence and a conceptual synthesis. American Journal of Orthopsychiatry, 58, 52-64.

Miscimarra, L., Stein, C., Millard, C., Kluge, A., Cartier, K., Freebairn, L., et al. (2007). Further evidence of pleiotropy influencing speech and language: Analysis of the DYX8 region. Human Heredity, 63, 47-58.

Mitsis, E. M., McKay, K. E., Schulz, K. P., Newcorn, J. H., \& Halperin, J. M. (2000). Parent-teacher concordance for DSM-IV attention-deficit/hyperactivity disorder in a clinic-referred sample. Journal of the American Academy of Child and Adolescent Psychiatry, 39, 308-313.

Morgan, A. E., Hynd, G. W., Riccio, C. A., \& Hall, J. (1996). Validity of DSM-IV ADHD predominantly inattentive and combined types: Relationship to previous DSM diagnoses/subtype differences. Journal of the American Academy of Child and Adolescent Psychiatry, 35, 325-333.

Newcomer, P. L., \& Hammill, D. D. (1997). Test of language development: Primary (TOLD-P:3). Austin: Pro-Ed.

Pennington, B. F. (2002). The development of psychopathology: Nature and nurture. New York: Guilford.

Pennington, B. F. (2006). From single to multiple deficit models of developmental disorders. Cognition, 101, 385-413.

Pennington, B. F., \& Lefly, D. L. (2001). Early reading development in children at family risk for dyslexia. Child Development, 72, 816-833.

Phares, V. (1997). Accuracy of informants: Do parents think that mother knows best? Journal of Abnormal Child Psychology, 25, $165-171$.

Raitano, N. A., Pennington, B. F., Tunick, R. A., Boada, R., \& Shriberg, L. D. (2004). Pre-literacy skills of subgroups of children with speech sound disorders. Journal of Child Psychology and Psychiatry, 45, 821-835.

Rucklidge, J. J., \& Tannock, R. (2002). Neuropsychological profiles of adolescents with ADHD: Effects of reading difficulties and gender. Journal of Child Psychology and Psychiatry, 43, 988-1003.

Scarborough, H. S. (1990). Very early language deficits in dyslexic children. Child Development, 61, 1728-1743.

Shriberg, L. D. (1993). Four new speech and prosody-voice measures for genetics research and other studies in developmental phonological disorders. Journal of Speech and Hearing Research, 36, 105-140.

Shriberg, L. D. (1994). Five subtypes of developmental phonological disorders. Clinics in Communication Disorders, 4, 38-53.

Shriberg, L. D. (2003). Diagnostic markers for child speech-sound disorders: Introductory comments. Clinical Linguistics \& Phonetics, 17, 501-505.

Shriberg, L. D., Allen, C. T., McSweeny, J. L., \& Wilson, D. L. (2001). PEPPER: Programs to examine phonetic and phonologic 
evaluation records. Madison: Waisman Center Research Computing Facility, University of Wisconsin-Madison.

Shriberg, L. D., Austin, D., Lewis, B. A., McSweeny, J. L., \& Wilson, D. L. (1997a). The percentage of consonants correct (PCC) metric: Extensions and reliability data. Journal of Speech, Language, and Hearing Research, 40, 708-722.

Shriberg, L. D., Austin, D., Lewis, B. A., McSweeny, J. L., \& Wilson, D. L. (1997b). The speech disorders classification system (SDCS): Extensions and lifespan reference data. Journal of Speech, Language, and Hearing Research, 40, 723-740.

Shriberg, L. D., Ballard, K. J., Tomblin, J. B., Duffy, J. R., Odell, K. H., \& Williams, C. A. (2006). Speech, prosody, and voice characteristics of a mother and daughter with a 7;13 translocation affecting FOXP2. Journal of Speech, Language, and Hearing Research, 49, 500-525.

Shriberg, L. D., Tomblin, J. B., \& McSweeny, J. L. (1999). Prevalence of speech delay in 6-year-old children and comorbidity with language impairment. Journal of Speech, Language, and Hearing Research, 42, 1461-1481.

SLI Consortium (2002). A genomewide scan identifies two novel loci involved in specific language impairment. American Journal of Human Genetics, 70, 384-398.

SLI Consortium (2004). Highly significant linkage to the SLI1 locus in an expanded sample of individuals affected by specific language impairment. American Journal of Human Genetics, 74, 1225-1238.

Smith, S. D., Pennington, B. F., Boada, R., \& Shriberg, L. D. (2005). Linkage of speech sound disorder to reading disability loci. Journal of Child Psychology and Psychiatry, 46, 1057-1066.
Snowling, M. J., Bishop, D. V. M., Stothard, S. E., Chipchase, B., \& Kaplan, C. (2006). Psychosocial outcomes at 15 years of children with a preschool history of speech-language impairment. Journal of Child Psychology and Psychiatry, 47, 759-765.

Stein, C. M., Millard, C., Kluge, A., Miscimarra, L. E., Cartier, K. C., Freebairn, L. A., et al. (2006). Speech sound disorder influenced by a locus in 15q14 region. Behavior Genetics, 36, 858-868.

Stein, C. M., Schick, J. H., Gerry Taylor, H., Shriberg, L. D., Millard, C., Kundtz-Kluge, A., et al. (2004). Pleiotropic effects of a chromosome 3 locus on speech-sound disorder and reading. American Journal of Human Genetics, 74, 283-297.

Tabachnick, B. G., \& Fidell, L. S. (2001). Using multivariate statistics (4th ed.). Boston: Allyn \& Bacon.

Tomblin, J. B., Records, N. L., \& Zhang, X. (1996). A system for the diagnosis of specific language impairment in kindergarten children. Journal of Speech and Hearing Research, 39, 1284-1294.

Werry, J. S., Elkind, G. S., \& Reeves, J. C. (1987). Attention deficit, conduct, oppositional, and anxiety disorders in children: III. Laboratory differences. Journal of Abnormal Child Psychology, $15,409-428$.

Willcutt, E. G., \& Pennington, B. F. (2000). Comorbidity of reading disability and attention-deficit/hyperactivity disorder: Differences by gender and subtype. Journal of Learning Disabilities, 33, 179-191.

Willcutt, E. G., Pennington, B. F., Smith, S. D., Cardon, L. R., Gayan, J., Knopik, V. S., et al. (2002). Quantitative trait locus for reading disability on chromosome $6 \mathrm{p}$ is pleiotropic for attention-deficit/ hyperactivity disorder. American Journal of Medical Genetics, 114, 260-268. 